\title{
Cross-sectional study to determine prevalence of significant liver fibrosis (F2-F4) in HIV/HCV co-infected patients: GRAFIHCO study C Tural ${ }^{1}$, E Ortega ${ }^{2}$, JA Pineda ${ }^{3}$, J Gonzalez-García ${ }^{4}$ L Griffa ${ }^{5}$, E Cabrero ${ }^{5}$ and A Burgos*5
}

\author{
Address: ${ }^{1}$ Hosp. Germans Trias y Pujol, Badalona, Spain, ${ }^{2}$ Hosp. Gral de Valencia, Valencia, Spain, ${ }^{3}$ Hosp. Univ. Valme, Sevilla, Spain, ${ }^{4}$ Hosp. \\ Univ. La Paz, Madrid, Spain and ${ }^{5}$ Abbott Laboratories SA, Madrid, Spain \\ * Corresponding author
}

from Ninth International Congress on Drug Therapy in HIV Infection

Glasgow, UK. 9-13 November 2008

Published: 10 November 2008

Journal of the International AIDS Society 2008, I I (SuppI I):P264 doi:I0.I I86/I758-2652-I I-SI-P264

This abstract is available from: http://www.jiasociety.org/content/I I/SI/P264

(c) 2008 Tural et al; licensee BioMed Central Ltd.

\section{Purpose of the study}

HIV/HCV co-infection is highly prevalent in Spain (50\%$60 \%$ ), and liver disease is the leading cause of morbidity and mortality among those individuals. Currently, liver biopsy is considered to be the gold standard for assessing significant liver fibrosis in the context of HCV-related liver disease. Multiple biopsies are impractical, costly, and could lead to infrequent but serious complications, however, non-invasive measures of liver fibrosis could therefore offer significant advantages. The objective of this cross-sectional study was to estimate the prevalence of significant liver fibrosis (SLF) in HIV/HCV patients in Spain using APRI and Forns index.

\section{Methods}

Patients are considered to have SLF if they have an APRI score $>1.5$ or an APRI score $<1.5$ but a Forns index score $>6.9$. Data from Fibroscan and biopsies were collected when available.

\section{Summary of results}

Results from 8,829 evaluable patients (78.3\% male) have been analyzed. Mean age $41.8 \pm 6.2$ years; $91.6 \%$ on antiretroviral treatment (ARVT). $57.9 \%$ of the patients were infected by HCV genotype 1, 20.5\% genotype 3, $16.1 \%$ genotype $4,3.2 \%$ genotype 2 , and $2.3 \%$ other genotypes. $30.6 \%$ of the patients were diagnosed of SLF according to the APRI/Forns algorithm. Nadir CD4 cell count was lower in patients with SLF than in those with- out SLF, median 159 cells/mcg vs. 191 cells/mcg, respectively $(\mathrm{p}<0.001)$; OR (for each 100 cell increase) $=0.92$ (95\% CI: 0.89-0.95). SLF was also more prevalent among male than among female patients, $32.7 \%$ vs. $23.2 \%$, respectively ( $<<0.001$ ); [OR 0.63 (95\% CI: 0.56-0.71)]. Results from biopsy and Fibroscan were collected in 1,701 and 1,879 patients, respectively. $53.3 \%$ of the patients had F2-F4 according to biopsy and 56.1\% according to Fibroscan $(>7 \mathrm{kPa})$. The positive predictive value of the algorithm according to biopsy results for SLF was $79.2 \%$ (95\% CI: 67.7-90.7).

\section{Conclusion}

An algorithm of biomarkers, including APRI and Forns index, identifies a significant proportion of HIV/HCV coinfected patients with F2-F4 liver fibrosis in daily clinical practice, although its performance is slightly lower than that reported in prior validation studies. Immunodepression and male sex are the main factors associated with significant fibrosis assessed by this procedure. 